\title{
WBSN based safe lifestyle: a case study of heartrate monitoring system
}

\author{
Vidhyotma, Jaiteg Singh \\ Chitkara University Institute of Engineering and Technology, Chitkara University, India
}

\begin{tabular}{|c|c|}
\hline Article Info & ABSTRACT \\
\hline $\begin{array}{l}\text { Keywords: } \\
\text { ECG } \\
\text { Embedded system } \\
\text { IoT } \\
\text { Smart healthcare } \\
\text { Wireless body sensor network } \\
\text { (WBSN) }\end{array}$ & $\begin{array}{l}\text { A Heart is the vital organ of the body. According to the "world health } \\
\text { statistics } 2017 \text { " by WHO, about } 460,000 \text { people die due to fatal heart attacks } \\
\text { every year. To reduce the death rate due to fatal heart attacks and } \\
\text { malfunctioning of the cardiovascular system, this paper proposed a Wireless } \\
\text { Body Sensor Network (WBSN) based, portable, easily affordable, } \\
\text { miniatured, accurate "Heartrate Monitoring System (HMS)". HMS can be } \\
\text { used to regularly examine the cardiac condition at home or hospital to avoid } \\
\text { or early detection of any serious condition. Heartrate Monitoring Algorithm } \\
\text { (HMA) was designed to observe the spread heartbeat spectrum and worked at } \\
\text { the backend of HMS. A case study was performed for forty healthy young } \\
\text { subjects. Each subject data was computed for sub }-3 S_{d}<\text { sub }<\text { sub }+ \\
3 S_{d} \text {. All subjects' } 99 \% \text { data lie in the custom range. The result produced by } \\
\text { HMS was the same as the previous medical record of subjects. } \\
\text { Copyright } \odot 2020 \text { Institute of Advanced Engineering and Science. } \\
\text { All rights reserved. }\end{array}$ \\
\hline \multicolumn{2}{|l|}{ Corresponding Author: } \\
\hline \multicolumn{2}{|c|}{$\begin{array}{l}\text { Vidhyotma, } \\
\text { Chitkara University Institute of Engineering and Technology, } \\
\text { Chitkara University, } \\
\text { Punjab, India. } \\
\text { Email: gandhi.vidhi25@gmail.com; vidhyotma.gandhi@ chitkara.edu.in. }\end{array}$} \\
\hline
\end{tabular}

\section{INTRODUCTION}

Health is the prime challenge for humanity around the globe. In the maintenance of health, a heart is one of the vital organs of the body. Heart and circulatory system make up a cardiovascular system which involves the circulation of blood to the organs, tissues and cells of the human body. Heart failure is a major health problem, faced by people all around the world. The cardiovascular diseases were rated the world's topmost cause of death by the World Health Organization (WHO) the number one cause of deaths across the world. According to the "world health statistics 2017" by WHO, about 460,000 people die due to heart attacks in a year and around half of these patients die within one hour as rushed to hospital [1]. The inexorable increase in death count would be poised in the near future. In conventional methods, one has to get hospitalized or rush to the hospital for monitoring the status of the heart and get treated accordingly which was time-consuming, expensive and out of reach for many people. Most of the time people neglect their health issues because of lack of time and financial support.

So, there is a need for a cost-effective, miniature and reliable heart health monitoring device which would be efficient and easy to use without any literacy boundaries. Wireless Body Sensor Network (WBSN) technology was developed to design smart healthcare systems. WBSN healthcare system was used to predict and treat the disorders and diseases at the earlier stage before it becomes difficult to handle or maybe a threat to human life [2]. It is expected that a smart healthcare system would increase the quality of health and decrease the risk to human life [3]. WBSN systems help to reduce the death rates due to delay in treatment in case of any type of sudden diseases strokes or accidents [2,3]. It was experiential that WBSN would increase the quality of health and decrease the cost of healthcare services [4]. For proper analyzation of ECG, 
an automatic method was developed with the combination of signal filtration and feature extraction. The digital bandpass filter was used for noise cancellation and the Yule-Walker or Short-Term Fourier Transform function was used for feature extraction [5, 6]. A generic model of the WBSN smart healthcare system is shown in Figure 1. To reduce the death rate due to fatal heart attacks and malfunctioning of the cardiovascular system, a portable, easily affordable, miniature, accurate cardiovascular healthcare monitoring system, "Heartrate Monitoring System (HMS)" was designed. HMS can be used to regularly examine the cardiac condition at home or hospital to avoid or early detection of any serious condition.

The main contributions of this paper are:

- $\quad$ Propose a WBSN based portable, low cost and accurate ECG monitoring and alarming device model.

- $\quad$ Design and implementation of accurate and time-efficient HMS.

- $\quad$ Evaluate the proposed WBSN based HMS model.

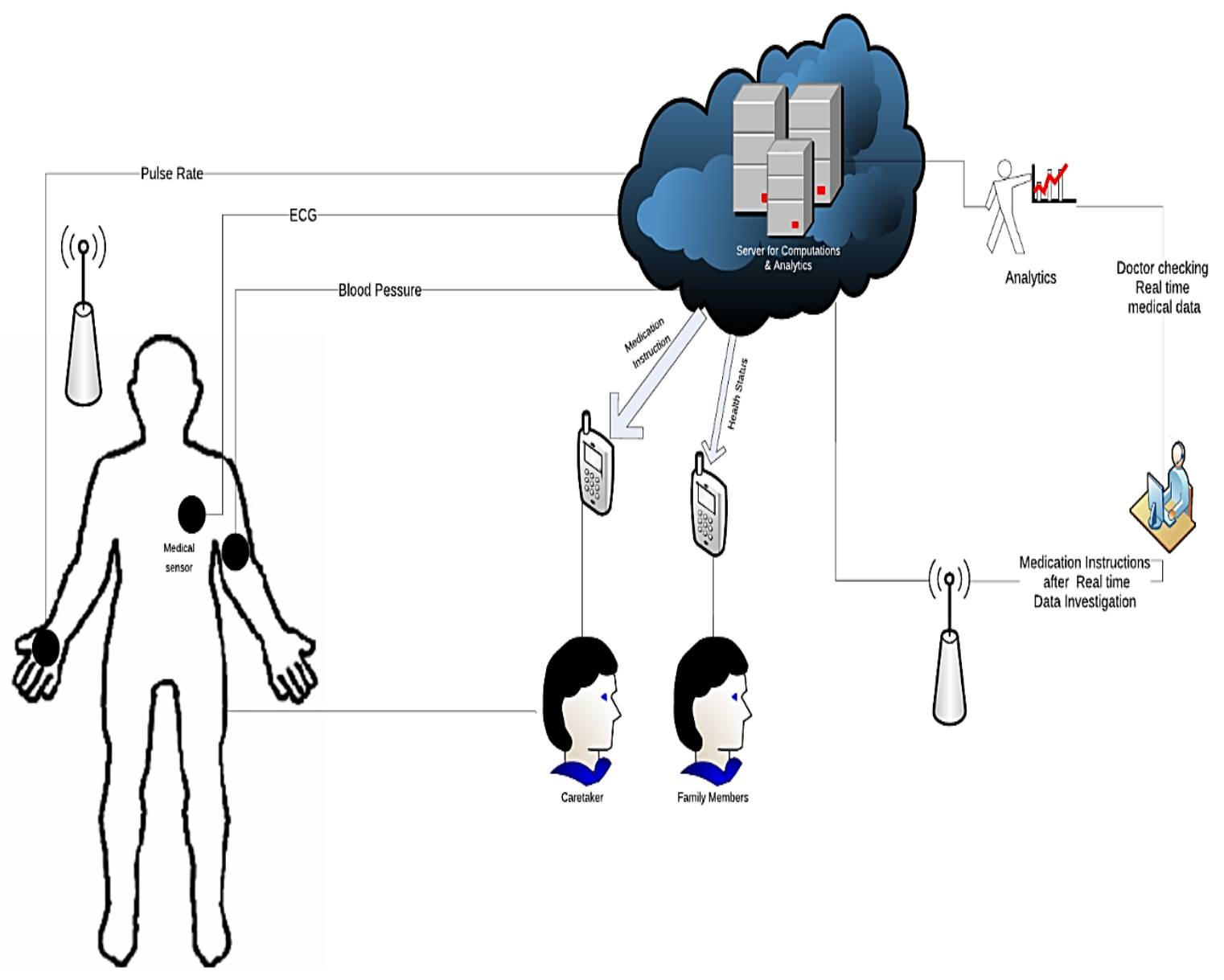

Figure 1. WBSN based smart healthcare system

\section{RESEARCH METHOD}

Many researchers worked to improve the heart healthcare systems for social welfare and tried to minimize human life risk. The technical details of adopted technologies for smart healthcare systems in the domain of heart diseases can be figured out from their publications given below:

- Valenzuela S. G. et al. (2010) presented a two-tier wireless system for non-critical convalescing patients, healthcare monitoring. The authors concluded that the system's performance would be affected by the placement of the WBSN coordinator node. The wrist position was amply favored for using as a temporary relay regardless of the patient's speed [7].

- $\quad$ Shang Hu et al. (2012) designed an energy efficient intelligent Body sensor network (iBoSen). It was a real-time wearable and lightweight electrocardiogram (ECG) monitoring and logging system. The smartphone was used as a gateway for visualizing and transmitting the ECG signals and other physiological signals like skin temperature, electroencephalogram (EEG) and respiration rate [8]. 
- Yeongjoon Gil et al. (2012) developed a lightweight, small size and low power operated the integrated system for measuring 4 signals: ECG, EEG, Photoplethysmography (PPG) and respiration. All sensors' data was verified in aspects of frequency responses and quantities. The system was tested for 31 years right-handed normal person sitting on a chair in a relaxed mood. The Blue tooth was used as communication. The authors suggested that power efficiency can be increased by using the Zigbee communication platform [2].

- $\quad$ Trobec R. et al. (2012) designed a multi-channel ECG sensor based on a respiration rate body sensor. The amplitude difference between two neighboring electrodes was compared respiration signal decoded from the air and temperature difference between mouth and nose. Seven persons with no medical record of age 45-50 were examined with over said respiration sensor. The authors identified the best position of electrodes for the respiration rate sensor by their experiments and confirm it a viable option. This sensor was not accurate for cardiovascular patients and also not energy efficient [9].

- Lee B. and Ouyang J. (2014) purposed an intelligent service model for patients to autonomously manage metabolic syndrome, the main cause of cardiovascular disease and brain stroke. Authors proposed an intellectualized service application algorithm which used two types of messages one was 'join' that was data request and the other was 'leave' that was data acknowledgment. The format used for a message was: i) Type of message ii) medical data iii) source address iv) destination address v) sequence number vi) length of payload vii) payload. A comprehensive assessment information protocol was used to provide an association between mutual diseases and menace [10].

- Miramonte R. et al. (2017) presented a wearable sensors based system to record heart rate, blood oxygen saturation level, etc. The PlalMos transmit data in a fixed environment using IEEE 802.15.4 and IEEE 802.11 with security mechanisms [11].

- $\quad$ Alshraideh H. et al. (2014) identified an efficient web-based cardiovascular disease detection system that can be used by patients anytime, anywhere through an android application. The data collected in January 1998 by the University of California at Irvina was used for experiments. The dataset of 452 instances contains 279 attributes and 16 arrhythmia classes. All WEKA (a data mining tool) algorithms were applied on this ECG data to check the best efficiency. The J48 algorithm provides maximum accuracy of $98.29 \%$ and NavieBayes' least accuracy of $75.06 \%$ [12].

The details of the bio-sensors and application area along with datasets used by the few researchers are summarized in Table 1. Presently computer-aided ambulance and intensive care of physiological parameters while traveling provides high latency and errors in data [1, 13, 14]. Remote monitoring and controlling of $\mathrm{BSN}$ is not time efficient $[15,16]$. In the last two decades, lots of prototypes and research projects have been industrialized and very well utilized the supportive technologies to achieve certain requirements of these systems. The detail of a few projects is given in Table 2 . In this study, a time-efficient heartrate monitoring algorithm (HMA) was designed to timely recognize any abnormality in ECG data.

Table 1. Application area and used data in the last decade of literature

\begin{tabular}{|c|c|c|c|c|c|c|c|c|c|c|c|c|}
\hline \multirow[b]{2}{*}{ Reference } & \multicolumn{8}{|c|}{ Bio Sensors } & \multicolumn{3}{|c|}{$\begin{array}{c}\text { Applications } \\
\text { Area }\end{array}$} & Data \\
\hline & 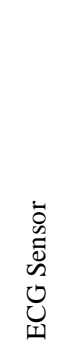 & $\begin{array}{l}\ddot{0} \\
0 \\
0 \\
\text { D } \\
0 \\
0 \\
\text { II }\end{array}$ & 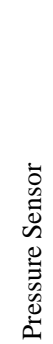 & 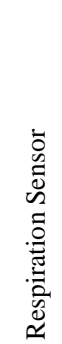 & $\begin{array}{l}\ddot{0} \\
0 \\
0 \\
\infty \\
\infty \\
\infty \\
\infty\end{array}$ & 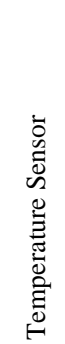 & 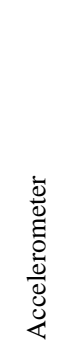 & 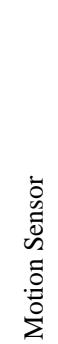 & 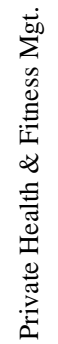 & 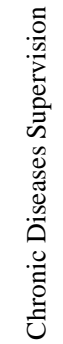 & 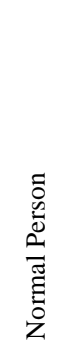 & Experimental Data \\
\hline$[17]$ & $\checkmark$ & & & & & & & $\checkmark$ & $\sqrt{ }$ & & & Synthetic \\
\hline [7] & $\checkmark$ & & & & & & $\checkmark$ & & & & $\checkmark$ & $\begin{array}{l}1 \text { person (4times in different } \\
\text { directions) }\end{array}$ \\
\hline [8] & $\checkmark$ & $\checkmark$ & & $\checkmark$ & & $\checkmark$ & & & & $\checkmark$ & $\checkmark$ & Synthetic data simulation \\
\hline [2] & $\checkmark$ & $\checkmark$ & $\checkmark$ & & & & & & & & $\checkmark$ & 1 person 31 -year old \\
\hline [9] & $\checkmark$ & & & $\checkmark$ & & & & & & & $\checkmark$ & $5 \mathrm{~F}+2 \mathrm{M}(45-50$ year $)$ \\
\hline [10] & & & & & $\checkmark$ & & & & & & & Virtual 1000 patients' sample \\
\hline
\end{tabular}


Table 2. Technical comparison of cardiovascular based research projects

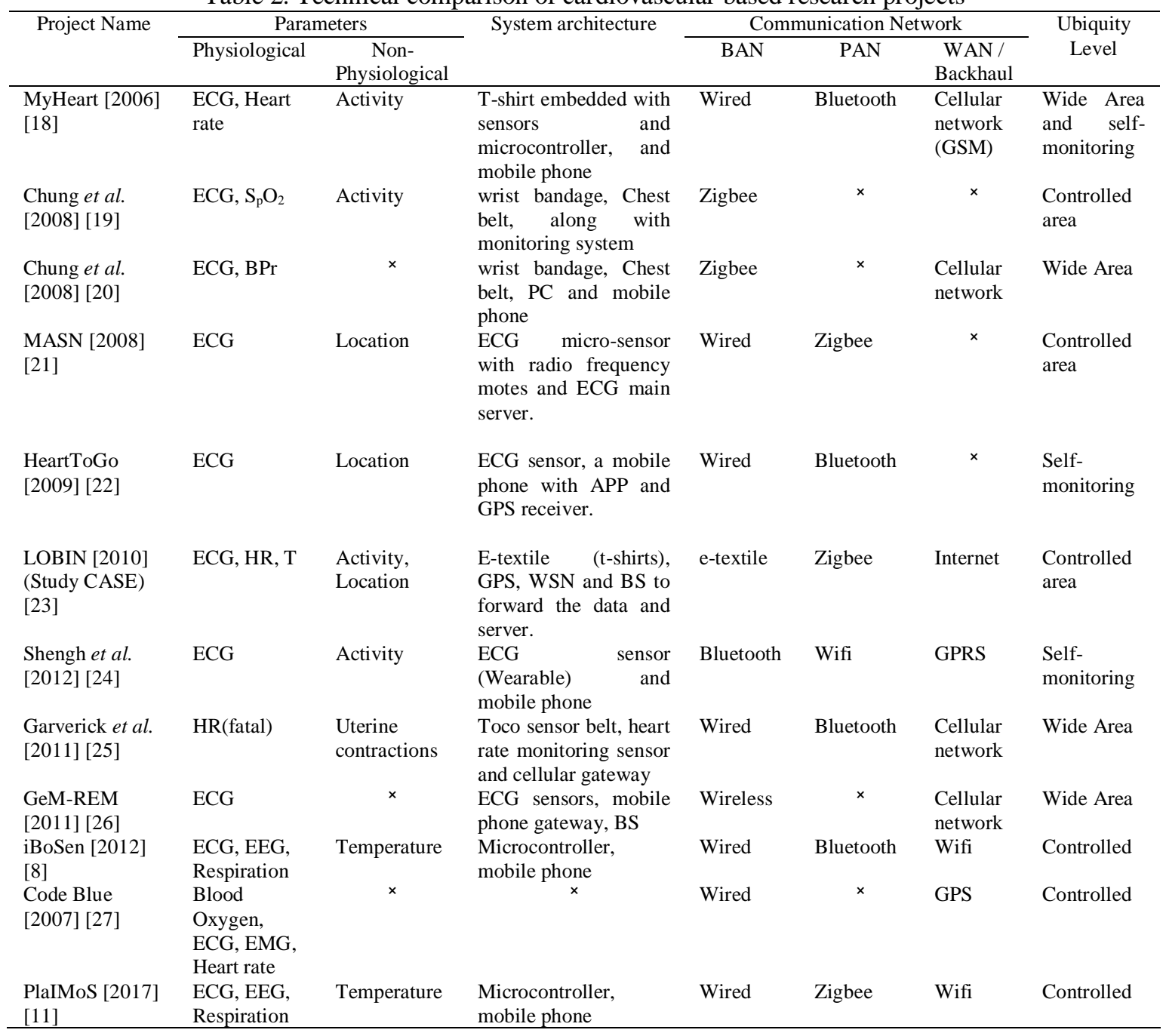

\section{PROPOSED MODEL}

This paper proposed an HMS system to provide better healthcare services to the society with the help of a device. The device was made by using raspberry pi attached with a couple of sensors. One can eventually monitor the cardio pulses in numerical (QT intervals values) as well as the graphical form before and after the workout (walking/jogging and/or pushups). The HMS system was synchronized with the pulses of the heart through sensors. The fitness of the heart was computed by the spread pattern and dispersion of recorded ECG data. The ECG data were recorded for pre and post workout conditions for precautionary measures to avoid any mishappenings.

Heartrate Monitoring System (HMS): HMS was designed to check the cardiovascular status at home/hospital/gym. It was consisting of raspberry pi B+ (Microcontroller Board (MCB)), 3-channel ECG sensor, 3-axis accelerometer, ultrasonic sensor and communication protocol. The global cloud data center was used to store data. Analytics were performed on the local server and cloud to auto recognize and alarm the emergency conditions. The block diagram of HMS is shown in Figure 2. To make HMS energy efficient, cooperative sensor interaction and centralized data abstraction mode were adopted [28]. For energy efficiency, the data was buffered for 1000 seconds before communication. The sampling frequency was 53.2 Hz. The detailed architecture is shown in Figure 3. 


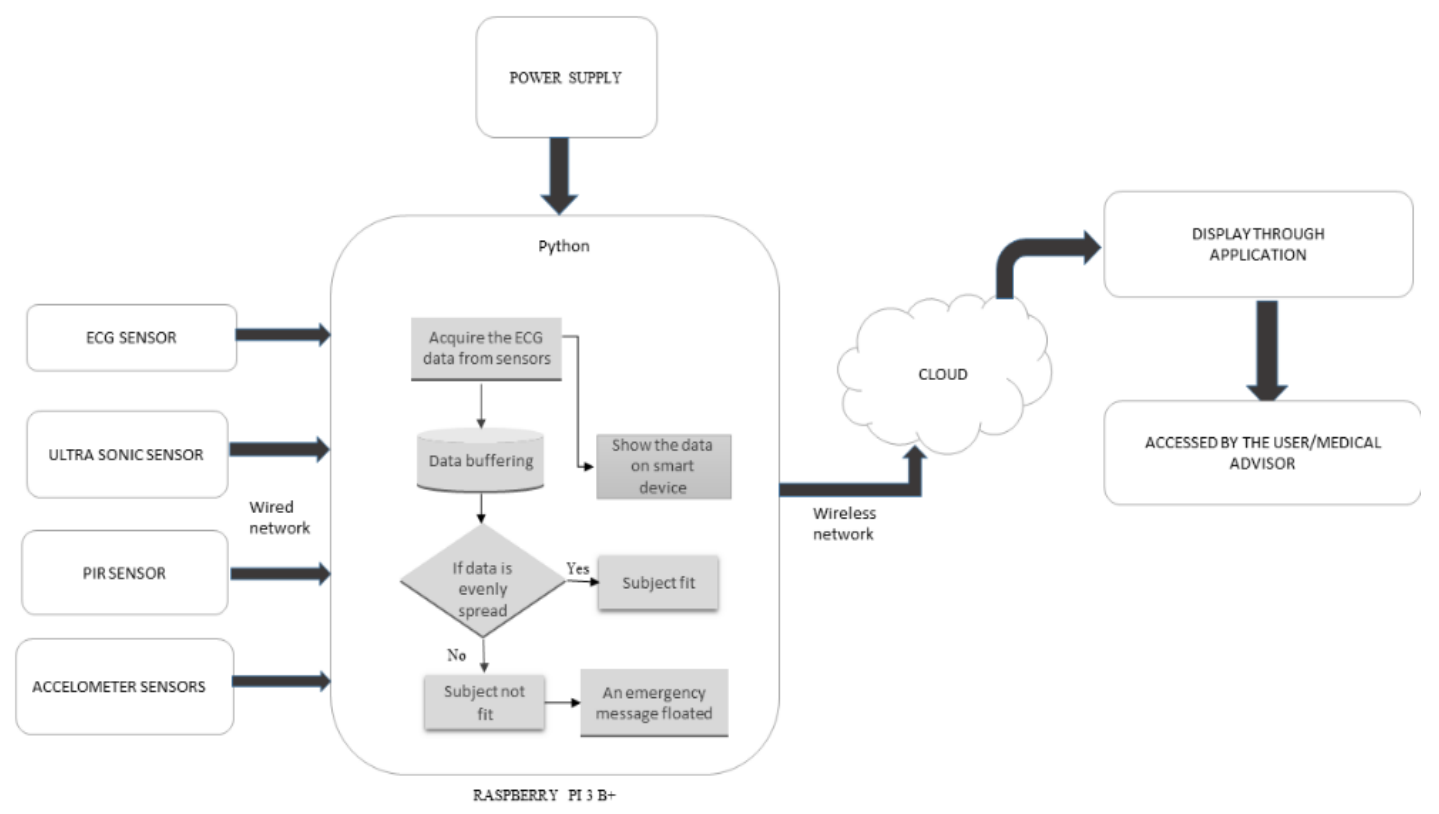

Figure 2. Block diagram of HMS

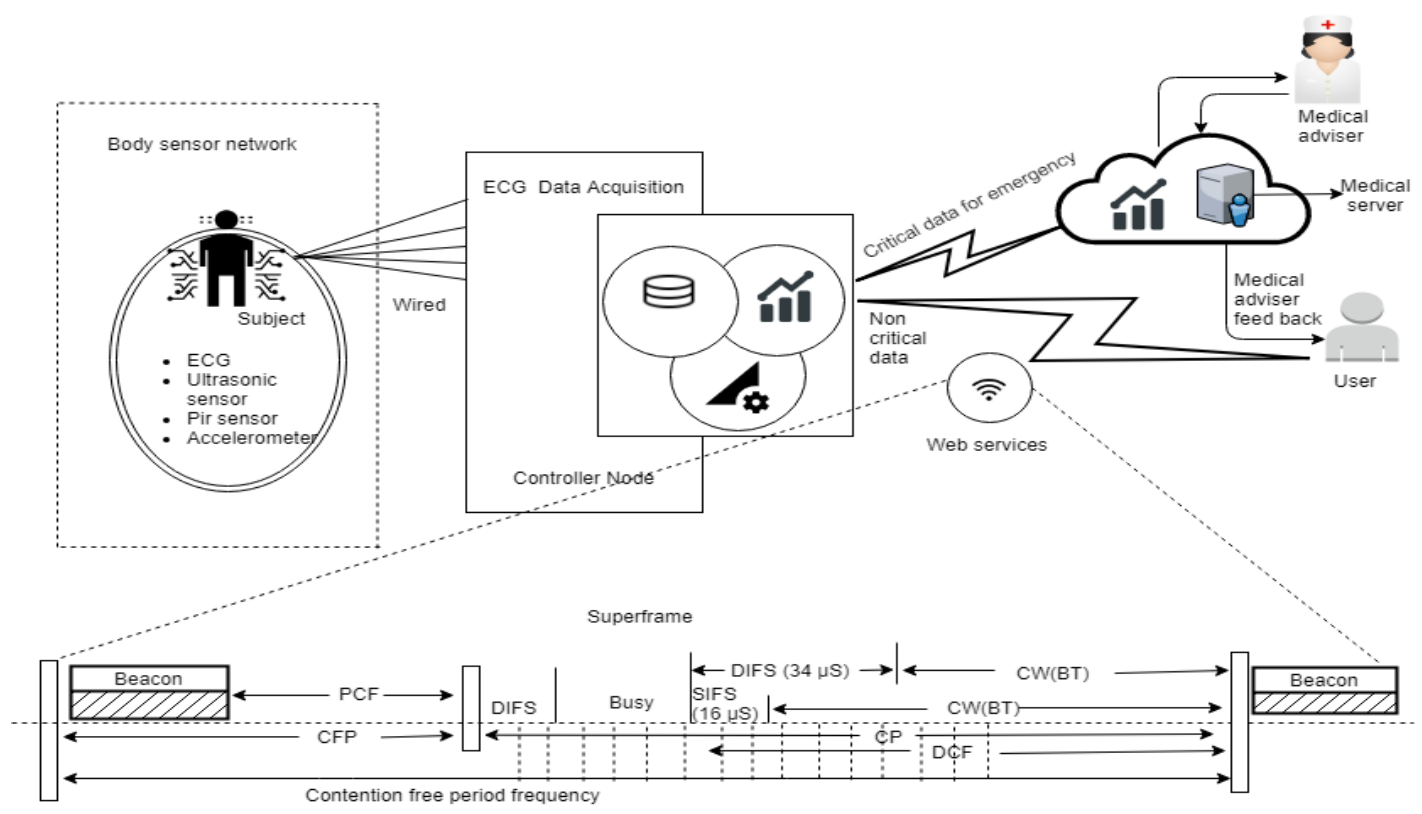

Figure 3. HMS architecture

\section{METHODOLOGY}

The experiment was performed on forty healthy teenagers (subjects) without any earlier medical records related to cardiovascular diseases. The data were recorded for each subject for pre and post workout conditions. Medical records, gender and age of subjects are given in Table 3. ECG data were recorded before and after ten minutes of workout. HMA was designed to work at the backend of HMS for the computations. The HMA algorithm is given in the next section. The HMA algorithm was applied to both (pre and post) data to check the heart health fitness of the subject. If the heart rate data was evenly spread then the person was declared as fit. If the heart rate data contained any outliers, the person's heart rate data was not in the custom range. 
Table 3. Experimental Subject Details

\begin{tabular}{cccc}
\hline Medical record & No. of subjects & Gender & Age \\
\hline NMR & 20 & Male & $18-20$ \\
NMR & 20 & Female & $19-20$ \\
\hline
\end{tabular}

NMR: No medical record

The first data was recorded at a rest position for 250 seconds. The second data was recorded after 5 minutes pause during the exercise (jogging or pushups). Two measurements, each 250 seconds long, were obtained from forty volunteers. All recorded data from the individual electrode were instantly examined for the good quality of the signal. In the case of malfunctioning in measurement, for instance, poor contact of an electrode, the data acquisition was restarted after solving the cause of the disturbance. The recorded heart rate data were saved for the next processing. No effort was made to disregard any measurements; so, some measurements have seldom arrhythmic events. All the measurements were obtained during our study at Chitkara University. ECG data range varies from person to person, so the individual person data was analyzed. To find out the heart rate data abnormalities in the recorded data, the standard deviation was calculated using (1).

$$
S_{d}=\sqrt{\frac{\sum(\overline{s u b}-s u b)^{2}}{n}}
$$

\section{PERFORMANCE EVALUATION}

Data of forty subjects were evaluated to measure the even spreadness and dispersion around the mean of a recorded data set. Even single outlier ECG data can raise the standard deviation value, which results in the abnormal heart condition. To check any distortion in recorded ECG data, the HMA algorithm was used and resulted as $99 \%$ data lie in proper intervals for subject 1.

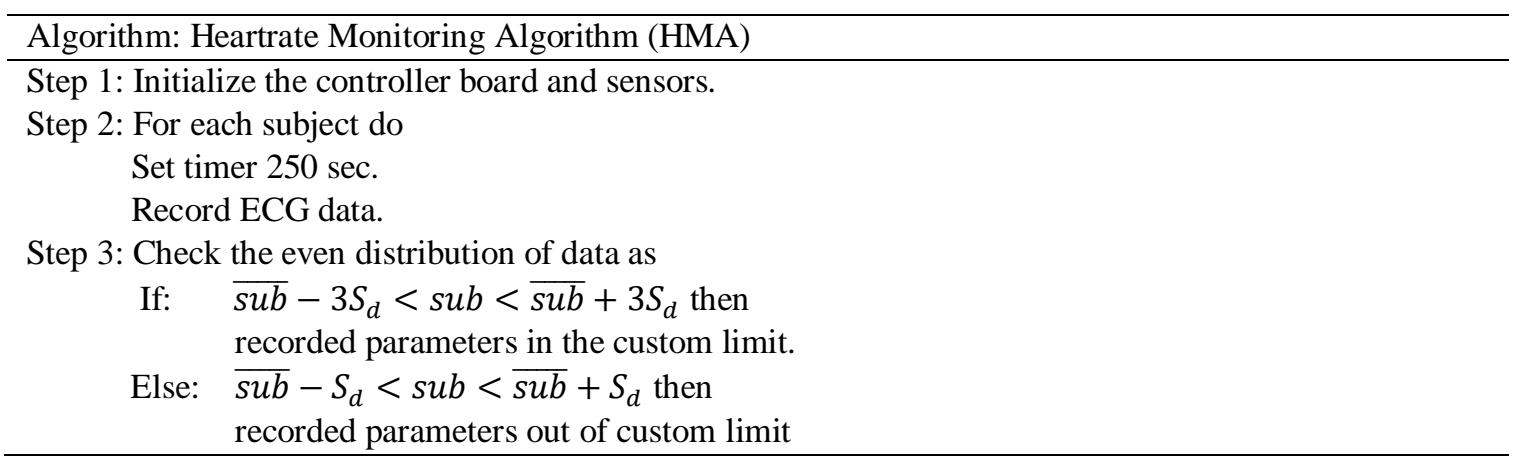

\section{RESULTS AND DISCUSSION}

Figures 4 and 5 are showing the heart rates of a few subjects at rest and during exercises respectively. It was observed that subject 1 data QT intervals varied between 263 to 428 , subject 2 data ranges from 275 to 486 . Whereas subject 3 data minimum value of QT intervals was 67 and the maximum value was 498 and subject 4 values lie in 28 to 545 QT intervals. According to their previous medical record, all were healthy persons. The results show that the heart rate beats vary subject to subject [27]. To check the spread heart rate spectrum, the variance of each subject heart rate was calculated as shown in Figure 6 and Table 4. The standard deviation was calculated to check the dispersion of data around the mean of the data set of each subject. The error line shown in Figure 6 was based on a five percent deviation that is in custom range. The heart rate data recorded by HMS and the results shown in Figure 6 and Table 4, all subjects are healthy as they were having a previous medical record. 


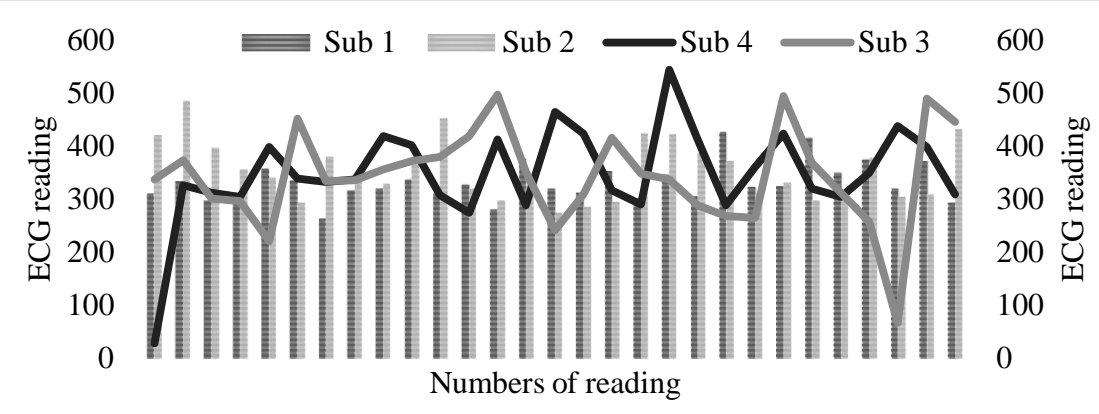

Figure 4. Heart rate at rest

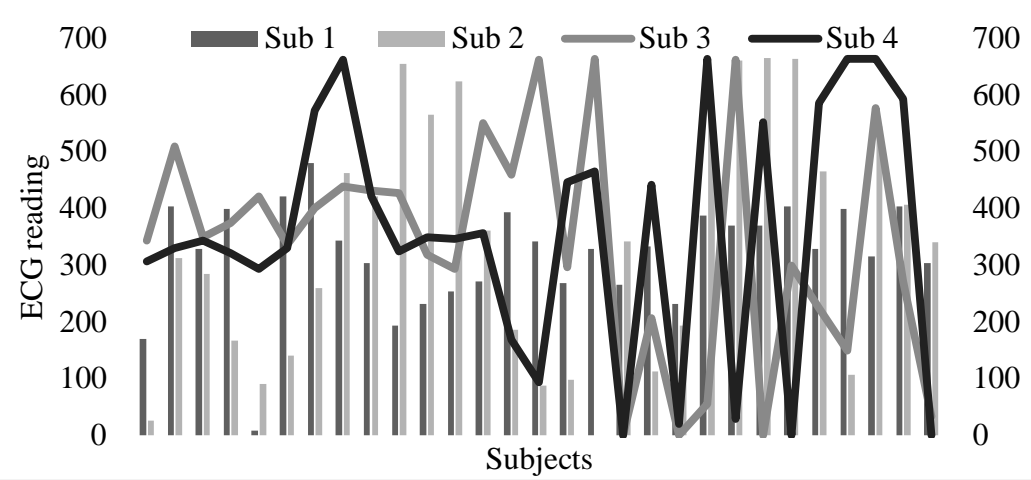

Figure 5. Heart rate during exercise

Table 4. Standard deviation $\left(S_{d}\right)$, mean, variance and median of four subjects' data

\begin{tabular}{cllllllll}
\hline & \multicolumn{3}{c}{ Exercise } & \multicolumn{4}{c}{ Rest } \\
\cline { 2 - 9 } & Sub 1 & Sub 2 & Sub 3 & Sub 4 & Sub 1 & Sub 2 & Sub 3 & Sub 4 \\
\hline$S_{d}$ & 92.87 & 216.51 & 197.63 & 217.03 & 37.21 & 55.64 & 91.86 & 89.32 \\
Mean & 319.06 & 338.59 & 336.34 & 356.828 & 331.79 & 354.28 & 343.034 & 349.59 \\
Var & 8932.2 & 46880.8 & 39055.31 & 47101.1 & 1384.4 & 3095.4 & 8438.11 & 7978.2 \\
Median & 329 & 341 & 343 & 346 & 324 & 341 & 337 & 337 \\
\hline
\end{tabular}

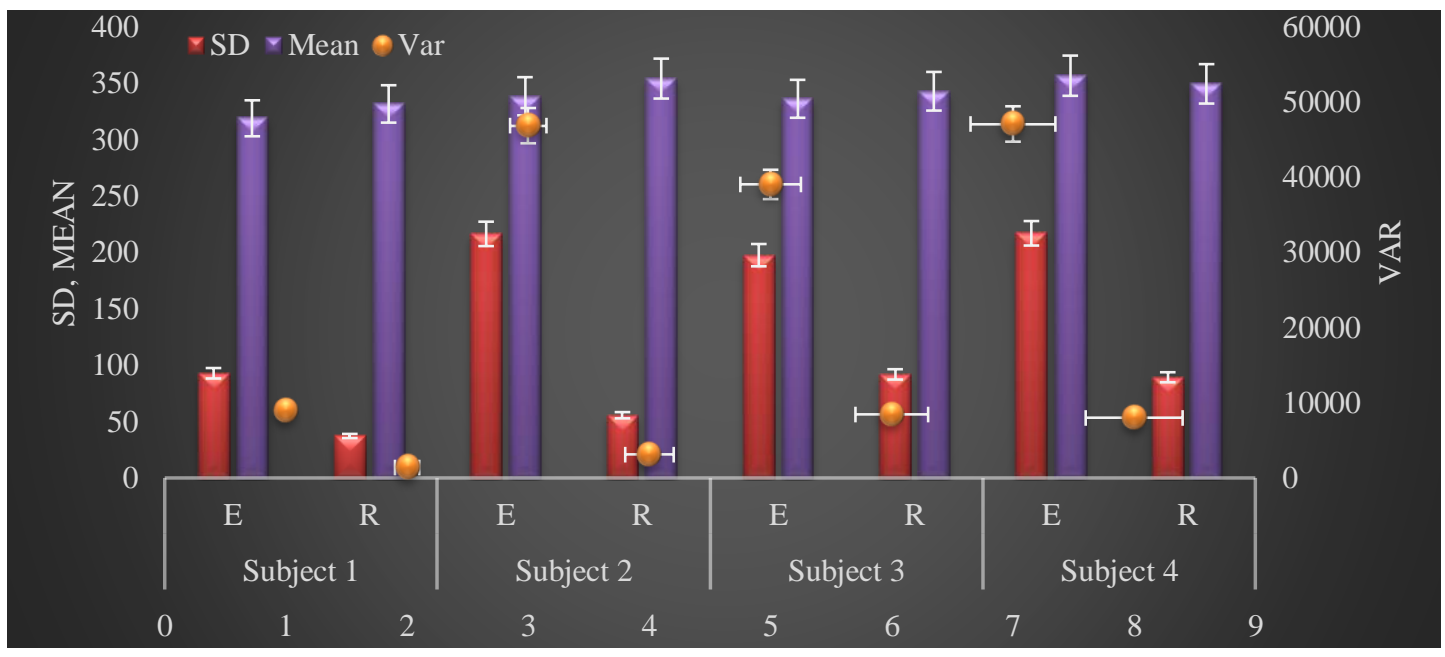

Figure 6. Standard deviation, mean and varience of four subjects' data 


\section{CONCLUSION}

The WBSN proved very helpful in a safe lifestyle. To reduce the death rate due to congestive heart failure, a WBSN based HMS system was designed. HMS can be used to regularly examine the cardiac condition at home or hospital to avoid or early detection of any serious condition. HMA was designed to observe the spread heart rate spectrum and outliers available in the recorded data. The existence of outliers in recorded data represents the abnormality of heart rate. The system was validated with the case study of forty healthy young subjects. The results produced by HMS were, each subjects' $99 \%$ data was in the custom range and all subjects were healthy as shown in their previous medical records.

\section{REFERENCES}

[1] “WHO," WHO, 2015. [Online]. Available: http://www.who.int/healthinfo/en.

[2] Y. Gil, W. Wu, and J. Lee, "A Synchronous Multi-Body Sensor Platform in a Wireless Body Sensor Network: Design and Implementation," Sensors, vol. 12, pp. 10381-10394, 2012.

[3] B. P. L. Lo, S. Thiemjarus, R. King, and G. Yang, "Body Sensor Network-A Wireless Sensor Platform for Pervasive Healthcare Monitoring," Adjun. Proc. 3rd Int. Conf. Pervasive Comput. (PERVASIVE'05, pp. 77-80, 2005.

[4] C. E. Koop et al., "Future Delivery of Health Care: Cybercare," IEEE Eng. Med. Biol. Mag., vol. 27, no. 6, pp. 29-38, Nov. 2008.

[5] V. Gupta and M. Mittal, "R-Peak Detection in ECG Signal using Yule-Walker and Principal Component Analysis," IETE J. Res., 2019.

[6] V. Gupta and M. Mittal, "QRS Complex Detection using STFT, Chaos Analysis, and PCA in Standard and RealTime ECG Databases,” J. Inst. Eng. Ser. B, Mar. 2019.

[7] S. González-Valenzuela and M. Chen, "Evaluation of Wireless Body Area Sensor Placement for Mobility Support in Healthcare Monitoring Systems," Ad Hoc Networks, pp. 384-399, 2010.

[8] S. Hu, H. Wei, Y. Chen, and J. Tan, "A Real-Time Cardiac Arrhythmia Classification System with Wearable Sensor Networks," Sensors, vol. 12, no. 12, pp. 12844-12869, Sep. 2012.

[9] R. Trobec, A. Rashkovska, and V. Avbelj, "Two Proximal Skin Electrodes-A Respiration Rate Body Sensor," Sensors (Switzerland), vol. 12, no. 10, pp. 13813-13828, 2012.

[10] B. M. Lee and J. Ouyang, "Intelligent Healthcare Service by using Collaborations between IoT Personal Health Devices," Int. J. Bio-Science Bio-Technology, vol. 6, no. 1, pp. 155-164, 2014.

[11] R. Miramontes et al., "PlaIMoS: A Remote Mobile Healthcare Platform to Monitor Cardiovascular and Respiratory Variables," Sensors (Switzerland), vol. 17, no. 1, pp. 1-24, 2017.

[12] H. B. Hussam Alshraideh, Mwaffaq Otoom, and Aseel Al-Araida, "A Web Based Cardiovascular Disease Detection System," in International Conference on Ubiquitous Computing and Ambient Intelligence, pp. 243-250, 2014.

[13] E. Jovanov, A. Milenkovic, C. Otto, and P. C. de Groen, "A Wireless Body Area Network of Intelligent Motion Sensors for Computer Assisted Physical Rehabilitation," J. Neuroeng. Rehabil., vol. 2, no. 1, p. 6, Mar. 2005.

[14] G. Zhao et al., "Exploration and Implementation of a Pre-Impact Fall Recognition Method Based on an Inertial Body Sensor Network," Sensors (Switzerland), vol. 12, no. 11, pp. 15338-15355, 2012.

[15] S. V. B. Peddi, P. Kuhad, A. Yassine, P. Pouladzadeh, S. Shirmohammadi, and A. A. N. Shirehjini, "An Intelligent Cloud-Based Data Processing Broker for Mobile E-Health Multimedia Applications," Futur. Gener. Comput. Syst., vol. 66, pp. 71-86, 2017.

[16] S. R. Moosavi et al., "End-to-end Security Scheme for Mobility Enabled Healthcare Internet of Things," Futur. Gener. Comput. Syst., vol. 64, pp. 108-124, 2016.

[17] A. Milenković, C. Otto, and E. Jovanov, "Wireless Sensor Networks for Personal Health Monitoring: Issues and an Implementation," Comput. Commun., vol. 29, no. 13-14, pp. 2521-2533, 2006.

[18] J. Habetha, "The MyHeart Project-Fighting Cardiovascular Diseases by Prevention and Early Diagnosis," in Annual International Conference of the IEEE Engineering in Medicine and Biology-Proceedings, pp. 6746-6749, 2006.

[19] W.-Y. Chung, Y.-D. Lee, and S.-J. Jung, "A Wireless Sensor Network Compatible Wearable U-Healthcare Monitoring System using Integrated ECG, Accelerometer and SpO2," in 2008 30th Annual International Conference of the IEEE Engineering in Medicine and Biology Society, pp. 1529-1532, 2008.

[20] W.-Y. Chung, S.-C. Lee, and S.-H. Toh, "WSN Based Mobile U-Healthcare System with ECG, Blood Pressure Measurement Function," in 2008 30th Annual International Conference of the IEEE Engineering in Medicine and Biology Society, pp. 1533-1536, 2008.

[21] F. Hu, M. Jiang, L. Celentano, and Y. Xiao, "Robust Medical Ad Hoc Sensor Networks (MASN) with WaveletBased ECG Data Mining," Ad Hoc Networks, vol. 6, no. 7, pp. 986-1012, Sep. 2008.

[22] Z. Jin, J. Oresko, S. Huang, and A. C. Cheng, "HeartToGo: A Personalized Medicine Technology for Cardiovascular Disease Prevention and Detection," in 2009 IEEE/NIH Life Science Systems and Applications Workshop, LiSSA 2009, pp. 80-83, 2009.

[23] G. López, V. Custodio, and J. I. Moreno, "LOBIN: E-Textile and Wireless-Sensor-Network-Based Platform for Healthcare Monitoring in Future Hospital Environments," IEEE Trans. Inf. Technol. Biomed., vol. 14, no. 6, pp. 1446-1458, Nov. 2010. 
[24] Y. C. and J. T. Sheng Hu, Hongxing wie, "A Real Time Cardiac Arrhythmia Classification System with Wearable Sensor Networks.pdf," Sensors, vol. 12, pp. 12844-12869, 2012.

[25] S. L. Garverick, H. Ghasemzadeh, M. Zurcher, M. Roham, and E. Saldivar, "Wireless Fetal Monitoring Device with Provisions for Multiple Births," in Proceedings - 2011 International Conference on Body Sensor Networks, BSN 2011, pp. 113-118, 2011.

[26] S. Nabar, A. Banerjee, S. K. S. Gupta, and R. Poovendran, "GeM-REM: Generative Model-Driven Resource Efficient ECG Monitoring in Body Sensor Networks,” in Proceedings - 2011 International Conference on Body Sensor Networks, BSN 2011, pp. 1-6, 2011.

[27] S. Somanchi, S. Adhikari, A. Lin, E. Eneva, and R. Ghani, "Early Prediction of Cardiac Arrest (Code Blue) using Electronic Medical Records," in Proceedings of the ACM SIGKDD International Conference on Knowledge Discovery and Data Mining, vol. 2015-August, pp. 2119-2126, 2015.

[28] G. F. Raffaele Gravina, Parastoo Alinia, Hassan Ghasemzadeh, "Multi-Sensor Fusion in Body Sensor Networks: State-of-the-Art and Research Challenges," Inf. Fusion, vol. 35, pp. 68-80, May 2017.

\section{BIOGRAPHIES OF AUTHORS}

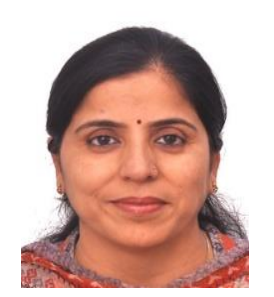

Vidhyotma is working in Chitkara University, Punjab as Assistant Professor, Research. She has worked as an Assistant Engineer in Punjab Communication Ltd. Mohali for 17 years and has teaching experience of 12 Years. She has completed her Masters in Computer Applications from Punjab Technical University, Jallandhar. M.Tech in CSE from Chitkara University, Punjab and is currently pursuing her Ph.D. in Computer Science Engineering from Chitkara University, Punjab.

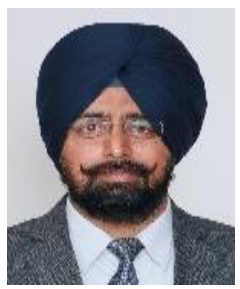

Dr Jaiteg holds a PhD in Computer Science and Engineering with 12 years of experience in Research, Development, Training, Academics at Institutes of Higher Technical Education. His areas of expertise are Software Engineering, Business Intelligence, Data and Opinion mining, Cartography, Curriculum design, Pedagogical Innovation \& Management. Areas of interest include Sustainable Software Engineering, Education Technology, Offline Navigation Systems and Cloud Computing. He had published more than 50 research articles in national and international journals. He had authored six books. 